\title{
Early-onset gastric cancers have a different molecular expression profile than conventional gastric cancers
}

\author{
Anya NA Milne ${ }^{1}$, Ralph Carvalho ${ }^{1}$, Folkert M Morsink ${ }^{1}$, Alex R Musler ${ }^{1}$, \\ Wendy WJ de Leng ${ }^{1}$, Ari Ristimäki ${ }^{2,3}$ and G Johan A Offerhaus ${ }^{1}$ \\ ${ }^{1}$ Department of Pathology, Academic Medical Center, Amsterdam, The Netherlands; ${ }^{2}$ Department of \\ Pathology, HUSLAB, Helsinki University Central Hospital, University of Helsinki, Helsinki, Finland \\ and ${ }^{3}$ Molecular and Cancer Biology Research Program, Biomedicum Helsinki, University of Helsinki, \\ Helsinki, Finland
}

\begin{abstract}
Many studies examine the molecular genetics of gastric cancer, but few look at young patients in particular and there is no comparison of molecular expression between early-onset gastric cancer ( $\leq 45$ years old) and conventional gastric cancers. Expression of cycloxygenase-2 (COX-2) is elevated in gastric adenocarcinomas compared to non-neoplastic mucosa, and in light of studies showing reduced risk of gastric cancer in nonsteroidal anti-inflammatory drug users, we have chosen to investigate the expression of COX-2 and related molecules in 113 early-onset gastric cancers and compare it with 91 conventional gastric cancers, using tissue microarrays. These markers include molecules known to be important in conventional gastric carcinogenesis, such as E-Cadherin, p53, COX-2, Trefoil Factor-1 (TFF1), $\beta$-catenin, p16 and c-myc; as well as molecules not yet described as being important in gastric cancer, such as the transcription factor c-jun, the COX-2 mRNA stabilizer HuR, and C/EBP- $\beta$, a transcription factor for COX-2. All markers showed a statistically significant difference between early-onset gastric cancers and conventional gastric cancers, using a $\chi^{2}$ test. In particular, early-onset gastric cancers displayed a COX-2 Low, TFF1-expressing phenotype, whereas COX-2 overexpression and loss of TFF1 was found in conventional cancers, and this difference between early-onset gastric cancers and conventional cancers remained statistically significant when adjusted for location and histology $(P<0.0001$ and $P=0.002$ respectively). We found that COX-2 overexpression correlates significantly with loss of TFF1 $(P=0.001)$, overexpression of C/EBP- $\beta(P<0.001)$ and cytoplasmic HuR $(P=0.016)$. COX-2 was significantly associated with $\mathrm{p53}$ positivity $(P=0.003)$. Abnormalities in E-Cadherin correlated significantly with diffuse phenotype, whereas high expression of COX-2, loss of TFF1 and overexpression of C/EBP- $\beta$ correlated with the intestinal phenotype. Our results provide further evidence that early-onset gastric cancer exhibits a distinctive expression profile that may have practical implications.
\end{abstract}

Modern Pathology (2006) 19, 564-572. doi:10.1038/modpathol.3800563; published online 10 February 2006

Keywords: early-onset gastric cancer; COX-2; TFF1

Gastric cancer is the second most common cause of cancer-related death in the world. ${ }^{1}$ It exists as two main histological types, diffuse and intestinal, as described by Laurén, ${ }^{2}$ and is thought to result from a combination of environmental factors and accumulation of specific genetic alterations, and in consequent, mainly affects older patients. Here, we investigate early-onset gastric cancer, which is

Correspondence: Dr ANA Milne, MD, Department of Pathology, L2-114, Academic Medical Center, Meibergdreef 9, 1105 AZ Amsterdam, The Netherlands.

E-mail: a.n.milne@amc.uva.nl

Received 3 November 2005; accepted 5 January 2006; published online 10 February 2006 defined as gastric cancer presenting at the age of 45 years or younger. Fewer than $10 \%$ of gastric cancer patients fall into the early-onset gastric cancer category $^{3}$ and it is postulated that genetic factors may be more important in these patients. ${ }^{4}$ Helicobacter pylori, a known causative agent in gastric carcinogenesis ${ }^{5-7}$ probably still plays a role in the development of gastric cancer in young patients; $;^{8,9}$ however, this is likely to involve a much smaller percentage of patients than that in the older age group.

Inherited gastric cancer predisposition syndromes account for approximately $10 \%$ of early-onset gastric cancers, yet even in these cases, the underlying genetic events are not always known. For 
example, alterations in the E-Cadherin gene have been associated with hereditary diffuse-type gastric cancer, ${ }^{10}$ but so far, only about $30 \%$ of all hereditary diffuse gastric cancer families carry CDH1 germline mutations. Thus, only a very small percentage of early-onset gastric cancers can be explained by E-Cadherin germline mutations, and the occurrence of gastric cancer in young patients remains largely unexplained.

Expression of cyclooxygenase-2 (COX-2) is elevated in gastric adenocarcinomas as compared to the non-neoplastic mucosa ${ }^{11,12}$ and in light of studies showing the reduced risk of gastric cancer in nonsteroidal anti-inflammatory drug users, ${ }^{13,14}$ investigating the difference in COX-2 expression between early-onset gastric cancer and conventional gastric cancer may have clinical implications. Thus, we have chosen to investigate the expression of COX-2 and related molecules in early-onset gastric cancer, and conventional gastric cancer using a tissue microarray approach. The COX-2 mRNA stabilizer $\mathrm{HuR}$ (Hu-Antigen R) and C/EBP- $\beta$ (CCAAT/Enhancer-Binding Protein- $\beta$ ), a transcription factor for COX-2, are also examined. C/EBP- $\beta$ is known to act not only as a transcription factor for COX-2, ${ }^{15}$ but also as a transcription factor in the downregulation of Trefoil Factor-1 (TFF1), ${ }^{16,17}$ loss of which has been independently shown to cause gastric adenomas. ${ }^{18}$ COX-2 overexpression has been found in these adenomas. ${ }^{19}$ Aberrant p53, known to be important in conventional gastric carcinogenesis, ${ }^{20}$ is also associated with increased levels of COX-2 expression. ${ }^{21}$ In addition, we examine p16, an important cell cycle regulator, which is lost in many gastric cancers. ${ }^{22}$

Wnt signalling may also be targeted through chemoprevention with nonsteroidal anti-inflammatory drugs, through a COX-2-independent mechanism $^{23}$ and it has been suggested that there is crosstalk between COX-2 and Wnt, ${ }^{24}$ thus c-myc, $\beta$-catenin and $\mathrm{c}^{-j u n^{25}}$ are also investigated in this study because of their involvement in the Wnt pathway. Finally, because of its importance in gastric cancer, ${ }^{10}$ the expression of E-Cadherin is included.

We aim to ascertain whether discernible differences exist in the expression of these markers, which would lend support to the theory that early-onset gastric cancers have distinct molecular characteristics from that of conventional gastric cancers. By the uncovering of the differences between gastric cancer in old and young patients, we are a step closer to understanding the pathogenesis of gastric cancer and elucidating new genes that may have prompted gastric cancer at an young age.

\section{Methods}

\section{Patients/Study Groups}

This research was carried out in accordance with the ethical guidelines of the research review committee of the Academic Medical Centre, Amsterdam. A total of 91 conventional gastric cancers ( $>45$ years old), diagnosed between 1993 and 2003, were obtained from the Academic Medical Centre, Amsterdam. A total of 113 cases of gastric carcinoma in patients under 45 years of age, 90\% diagnosed between 1994 and 2002 and 10\% diagnosed between 1980 and 1994, were obtained from 24 different institutions throughout the Netherlands through the nationwide database system, and from the Department of Pathology at the Jorvi Hospital (Espoo, Finland). This age cutoff was chosen in order to obtain enough cases to achieve meaningful result. The tumors were classified by an experienced gastrointestinal pathologist (GJAO) according to the Laurén classification as intestinal, diffuse or mixed gastric adenocarcinomas, and location was deduced from the pathological report (if available) as seen in Table 1.

\section{Tissue Microarray}

Tissue microarrays were constructed from formalinfixed and paraffin-embedded archive specimens. Three core biopsies $(0.6 \mathrm{~mm}$ cylinders $)$ were taken from histologically representative regions (including heterogeneous areas) of paraffin-embedded gastric tumors and arranged in a new recipient paraffin block (tissue array block), using a custom-built instrument (Beecher Instruments, Silver Spring, MD, USA) as also described previously. ${ }^{26}$ Normal gastric mucosa from each case was also included where available (69/204). Cores were arranged in two or three separate subdivisions together with insertion of liver, lymph node and kidney cores to assist analysis. A total of 189 cases were informative for all markers. In most cases, sections were stained immediately after cutting, but if stored, this was

Table 1 Clinicopathological characteristics

\begin{tabular}{|c|c|c|c|c|c|c|c|}
\hline \multirow[t]{2}{*}{ Age (years) } & \multicolumn{3}{|c|}{ Histology } & \multicolumn{3}{|c|}{ Location } & \multirow[t]{2}{*}{ Total } \\
\hline & Intestinal & Diffuse & Mixed & Cardia & Noncardia & Unknown & \\
\hline$\leq 45$ & 24 & 80 & 9 & 9 & 74 & 30 & 113 \\
\hline$>45$ & 49 & 31 & 11 & 49 & 42 & 0 & 91 \\
\hline
\end{tabular}


done so by wrapping in aluminum foil and freezing at $-20^{\circ} \mathrm{C}$ to prevent loss of antigenicity.

\section{Immunohistochemistry}

Sections $(4 \mu \mathrm{m})$ were deparaffinized and antigen retrieval was carried out by $10 \mathrm{~min}$ of boiling in $10 \mathrm{mM}$ Tris/1 mM EDTA (pH 9), except for c-myc where no antigen retrieval was used. Subsequently slides were immersed in $0.3 \%$ hydrogen peroxide in methanol for $30 \mathrm{~min}$ and nonspecific binding was blocked with $5 \%$ normal goat serum for $1 \mathrm{~h}$ at room temperature. The sections were incubated for $1 \mathrm{~h}$ (or overnight in the case of $\mathrm{C} / \mathrm{EBP}-\beta$ ) at room temperature with the following primary antibodies: p53 (monoclonal antibody combination of DO-7 and BP53-12, Neomarkers, Union City, CA, USA) 1:2000 dilution, E-Cadherin HECD-1 (Thamer) 1:2000 dilution; TFF1/pS2 (Dako) 1:1200 dilution, c-myc (Santa Cruz) 1:50, NCL c-jun (Nova Castra) 1:200, $\beta$-catenin (BD Biosciences, Alphen aan den Rijn, The Netherlands) 1:10 000 dilution, p16 $6^{\mathrm{INK} 4 \mathrm{~A}}$ (Neomarkers) 1:100 dilution, C/EBP- $\beta$ (H-7): sc-7962 (Santa Cruz Biotechnology, Santa Cruz, CA, USA) 1:50 dilution. The Ultravision antipolyvalent HRP detection system (Lab Vision Corp., Fremont, CA, USA) was used to visualize antibody binding sites with $3,3^{\prime}$-diaminobenzidine as a chromogen. DAB Plus was used for c-jun. Sections were counterstained with hematoxylin.

Immunohistochemistry for COX-2 was carried out as above with the following exceptions: antigen retrieval was carried out in $0.01 \mathrm{M}$ Na-citrate buffer (pH 6.0), followed by immersion in $0.6 \%$ hydrogen peroxide in methanol for $30 \mathrm{~min}$ and then in blocking solution $\left(0.01 \mathrm{M}\right.$ Tris, $0.1 \mathrm{M} \mathrm{MgCl}_{2}, 0.5 \%$ Tween-20, 1\% BSA, 5\% normal goat serum) for $1 \mathrm{~h}$. Incubation of the primary antibody was carried out using monoclonal COX-2 antibody at a dilution of 1:100 (Cayman Chemical Co., Ann Arbor, MI, USA) at $4^{\circ} \mathrm{C}$ overnight.

Immunohistochemistry for HuR was carried out as follows: specimens were deparaffinized and antigen retrieved using microwave oven $(4 \times 5 \mathrm{~min}$ in $700 \mathrm{~W}$ in $0.01 \mathrm{M} \mathrm{Na}$-citrate buffer (pH 6.0)). The slides were then immersed in $0.6 \%$ hydrogen peroxide in methanol for $30 \mathrm{~min}$ to block endogenous peroxidase activity, and in blocking solution (1:66 normal horse serum in PBS) for 15 min to block unspecific binding sites. Immunostaining for $\mathrm{HuR}$ was performed with monoclonal antibody $19 \mathrm{~F} 12,{ }^{27}$ in a dilution of 1:10000 $(1.0 \mu \mathrm{g} / \mathrm{ml})$ in PBS containing $0.1 \%$ sodium azide and $0.5 \%$ BSA, overnight at room temperature. The sections were then treated with biotinylated horse anti-mouse immunoglobulin (1:200; Vector Laboratories Inc., Burlingame, CA, USA) and avidin-biotin peroxidase complex (Vectastain ABComplex; Vector Laboratories) and peroxidase staining visualized with 3-amino-9ethylcarbazole (Sigma Chemical Co., St Louis, MO, USA). Sections were counterstained with Mayer's hematoxylin.

\section{Scoring}

Scoring of immunohistochemistry was carried out for $\mathrm{p} 53,{ }^{28} \mathrm{E}$-Cadherin, ${ }^{29} \mathrm{TFF},{ }^{30} \beta$-Catenin, ${ }^{31} \mathrm{COX}-2,{ }^{32}$ p16, ${ }^{33} \mathrm{HuR}^{34} \mathrm{C} / \mathrm{EBP}-\beta,{ }^{15} \mathrm{C}^{3} \mathrm{myc}^{35}$ and $\mathrm{c}$-jun, as shown in Table 2 .

The overall score of the tumor was the highest score found. No slides showed increased negative staining around the edges. Unequivocal cases were scored by one observer (ANAM). All other cases (approximately 30\%) were assessed by two observers (ANAM and GJAO) and agreement reached using a multiheaded microscope. Cases where the staining was not obviously abnormal were placed in the normal category. All tissue microarray slides were examined carefully, and results documented irrespective of scoring system. Subsequently, various scoring systems in the literature were assessed in order to choose a system that accurately reflected the results obtained and all cases were re-examined based on this scoring system. This was not the case, however, with more established scoring systems such as with p53, E-Cadherin, COX-2, and

Table 2 Scoring of immunohistochemistry

\begin{tabular}{ll}
\hline Molecule & Criteria for abnormal scoring \\
\hline p53 & Strong nuclear staining in $>30 \%$ of cells \\
E-Cadherin & Absence of membranous staining or presence of clumpy cytoplasmic localization \\
TFF & Absence of staining in $95 \%$ of tumor cells \\
$\beta$-Catenin & Nuclear staining accompanied by loss of membranous staining and increased cytoplasmic staining \\
COX-2 & 0-no staining; 1-very weak diffuse cytoplasmic staining \\
& 2--moderate-to-strong granular cytoplasmic staining in 10-50\% \\
& C->50\% of tumor cells with strong intensity \\
p16 & Categories 0 and 1 were COX-2 Low, categories 2 and 3 were COX-2 High \\
c-myc & Nuclear staining in $>5 \%$ \\
c-jun & Nuclear staining in $>5 \%$ \\
HuR & The presence of staining was scored separately as positive or negative for the nucleus and cytoplasm of tumor cells \\
C/EBP- $\beta$ & Nuclear staining $>25 \%$ of cells
\end{tabular}


$\beta$-Catenin, which were scored according to wellestablished systems that are familiar to the authors.

\section{Statistical Analysis}

The SPSS 11.5 software package was used for statistical analysis. A $\chi^{2}$ test was applied to the groups of gastric cancer to determine whether the differences found between antibodies were statistically significant $(P<0.05)$. A binary logistic regression model was used to adjust for potential confounding factors such as location, histological type, age of blocks and the hospital from which the block was derived.

\section{Results}

Immunohistochemical results for early-onset gastric cancer, conventional gastric cancers and the combined results can be seen in Table 3 . Variation of staining between cores ranged from no cases in E-Cadherin and $\beta$-catenin to 4/204 cases in c-myc, c-jun, p16, TFF1, C/EBP- $\beta$. These cases had one or two cores that fell within the positive category and one or two that fell within the negative category. The most striking results as seen in Table 3 involve COX-2 and TFF1: the COX-2 High phenotype was present in $66 \%$ of conventional gastric carcinomas but in only $10 \%$ of early-onset gastric cancer conversely, loss of TFF1 was seen in $73 \%$ of conventional gastric cancers, but in only $39 \%$ of early-onset gastric cancers. In order to compare critically these results obtained for early-onset gastric cancer and conventional gastric cancers, we carried out $\chi^{2}$ analysis. In addition, we compared the groups by using a binary logistic regression model to examine whether the $\chi^{2}$ findings were significant once adjusted for location and histology. In this way, we compared the cancers with respect to anatomic location (adjusted for age and histology), and histology (adjusted for age and location). In addition, using the binary logistic regression model, we confirmed that neither the age of the blocks, nor the hospital from which they originated (thus possible variation in processing) significantly affected the results for any marker used.

\section{Correlation with Age, Location and Histology}

We can see from Table 4 that all markers showed a statistically significant difference between earlyonset gastric cancers and conventional gastric cancers. COX-2 showed much higher expression levels in conventional gastric cancer than in earlyonset gastric cancer, and loss of TFF1 was much more common in conventional gastric cancer than in early-onset gastric cancer. Furthermore, the difference found between the groups with COX-2 $(P<0.001)$ and TFF1 $(P<0.001)$ remained statistically significant when adjusted for location and histology $(P<0.0001$ and $P=0.002$, respectively).

In addition, we found that p53 positivity and p16 negativity correlated significantly with tumors of the cardia $(P=0.007$ and $P=0.016$ respectively), whereas c-jun positivity correlated with non-cardia tumors $(P=0.005)$. Abnormalities in E-Cadherin correlated significantly with the diffuse phenotype $(P<0.001)$, whereas COX-2 High, loss of TFF1 and overexpression of C/EBP- $\beta$ correlated with the intestinal phenotype $(P=0.008, P=0.04$, and $P=0.001$, respectively).

\section{Correlation between Molecular Markers}

Using a $\chi^{2}$ test, we found that COX-2 overexpression correlates significantly with loss of TFF1 $(P=0.001)$, overexpression of C/EBP- $\beta(P<0.001)$ and cytoplasmic (but not nuclear) HuR $(P=0.016)$. This phenotype can be seen in Figure 1. In addition COX-2 was significantly associated with p53 positivity

Table 3 Results of Immunohistochemistry

\begin{tabular}{|c|c|c|c|c|c|}
\hline \multirow[t]{2}{*}{ Antibodies } & \multicolumn{3}{|c|}{ Immunohistochemical findings ${ }^{\mathrm{a}}$} & \multicolumn{2}{|c|}{ Range of staining } \\
\hline & Age $>45$ years old & Age $<45$ years old & All Ages combined & Negative & Positive \\
\hline E-Cadherin & $37 \%$ abnormal & $52 \%$ abnormal & $45 \%$ positive & NR & NR \\
\hline$\beta$-catenin & $23 \%$ abnormal & $35 \%$ abnormal & $29 \%$ abnormal & NR & NR \\
\hline p53 & $48 \%$ positive & $31 \%$ positive & $42 \%$ positive & $0-10 \%$ & $50-90 \%$ \\
\hline $\mathrm{COX}-2$ & $66 \%$ High & 10\% High & 35\% High & NR & NR \\
\hline $\mathrm{TFF}$ & $73 \%$ negative & $39 \%$ negative & $55 \%$ negative & $0-1 \%$ & $25-99 \%$ \\
\hline HuR cytoplasmic & $28 \%$ positive & $13 \%$ positive & $34 \%$ positive & $0 \%$ & $15-100 \%$ \\
\hline HuR nuclear & $40 \%$ positive & $28 \%$ positive & $34 \%$ positive & $0 \%$ & $20-100 \%$ \\
\hline $\mathrm{c} / \operatorname{ebp} \beta$ & $80 \%$ positive & $67 \%$ positive & $73 \%$ positive & $0-9 \%$ & $35-100 \%$ \\
\hline p16 & $57 \%$ negative & $34 \%$ negative & $44 \%$ negative & $0-5 \%$ & $20-100 \%$ \\
\hline c-myc & $33 \%$ positive & $52 \%$ positive & $43 \%$ positive & $0-5 \%$ & $6-100 \%$ \\
\hline c-jun & $64 \%$ positive & $74 \%$ positive & $70 \%$ positive & $0-5 \%$ & $6-100 \%$ \\
\hline
\end{tabular}

${ }^{\mathrm{a}}$ To the nearest percent.

NR, not relevant. 
Table 4 Statistical analysis of immunohistochemical results

\begin{tabular}{|c|c|c|c|c|}
\hline \multirow[t]{3}{*}{ Antibodies } & \multicolumn{4}{|c|}{ Statistical significance } \\
\hline & \multirow{2}{*}{$\begin{array}{c}\chi^{2} \\
\text { Age }\end{array}$} & \multicolumn{3}{|c|}{ Binary logistic regression model } \\
\hline & & Age & Location & Histology \\
\hline E-Cadherin & $P=0.027$ & None & None & $P<0.001$ (CI 3.2-16.3) \\
\hline$\beta$-Catenin & $P=0.049$ & None & None & None \\
\hline p53 & $P=0.009$ & None & $P=0.004($ CI $0.2-0.7)$ & None \\
\hline COX-2 & $P<0.001$ & $P<0.001$ (CI 6.3-50.1) & None & $P=0.008$ (CI $0.1-0.7)$ \\
\hline TFF1 & $P<0.001$ & $P=0.001($ CI 1.7-7.6) & None & $P=0.04($ CI $0.2-0.97)$ \\
\hline HuR cytoplasmic & $P=0.005$ & None & None & None \\
\hline HuR nuclear & None & None & None & None \\
\hline $\mathrm{C} / \mathrm{EBP}-\beta$ & $P=0.033$ & None & None & $P=0.001($ CI $0.08-0.52)$ \\
\hline p16 & $P=0.001$ & None & $P=0.014($ CI 1.2-5.6) & None \\
\hline c-myc & $P=0.015$ & None & None & None \\
\hline c-jun & $P=0.08$ & None & $P=0.012($ CI 1.3-6.1) & None \\
\hline
\end{tabular}

$\chi^{2}$ test was carried out for each antibody vs age. The results displayed under the heading Binary logistic regression model are as follows: age, adjusted for location and histology; location, adjusted for age and histology; and histology (results for diffuse histology given) adjusted for age and location.

$(P=0.003)$. On the other hand, TFF1 did not correlate significantly with cytoplasmic HuR, nuclear HuR or p16, and neither did the correlation with C/EBP- $\beta$ reach statistical significance $(P=0.059)$.

Nuclear HuR correlated significantly with c-myc positivity $(P<0.001)$ but did not correlate with C/EBP- $\beta$.

No correlation was found between abnormal $\beta$ catenin and c-myc or c-jun positivity.

\section{Discussion}

Many studies examine the molecular genetics of gastric cancer in general, but very few look at young patients in particular and there is no comparison of molecular expression between early-onset gastric cancers and conventional gastric cancers. Here, we examine a large group of 204 gastric cancers, approximately half of which are early-onset gastric cancers, and compare the expression of 10 molecular markers in early-onset gastric cancer and conventional gastric cancers.

Expression of COX-2 is elevated in gastric adenocarcinomas as compared to the non-neoplastic mucosa. ${ }^{11}$ Interestingly, in this study we find that COX-2 expression varies significantly between early-onset gastric cancers and conventional cancers, with COX-2 overexpression occurring rarely in early-onset gastric cancers. In light of studies showing the reduced risk of gastric cancer in nonsteroidal anti-inflammatory drug users, ${ }^{13,14}$ our results may have clinical implications, as they suggest that this reduced risk may apply only to gastric cancers in older patients as COX-2 does not appear to play an important role in early-onset gastric cancer. It also implies that genetic changes typical for conventional tumors more readily induce
COX-2 expression than those associated with earlyonset gastric cancer.

HuR is a member of the Hu family of ARE-binding proteins involved in the regulation of mRNA turnover, ${ }^{36}$ which shuttles between the nucleus and the cytoplasm. In this study, we find that cytoplasmic HuR correlates with COX-2 expression in gastric cancer. COX-2 mRNA is known to contain HuRbinding AREs in its $3^{\prime}$ untranslated region, and HuR has been associated with prognosis and COX-2 expression in human carcinomas. ${ }^{34,37}$

COX-2 is predominantly expressed in intestinal-type gastric carcinomas and its precursor lesions, ${ }^{38,39}$ as confirmed in this study. We also find that p53 immunopositivity (as a marker for loss of p53 function) is associated with increased level of COX-2 expression, as previously described. ${ }^{21}$ These results support the theory that there may be a direct link between the defective p53 pathway and elevated levels of COX-2 expression in cancer cells.

In addition to COX-2, we also find a significant difference in TFF1 expression between the earlyonset gastric cancers and the conventional cancers, with loss of TFF1 occurring less frequently in the early-onset gastric cancer group. TFF1 is synthesized and secreted by the normal stomach mucosa and by the gastrointestinal cells of injured tissues. The link between mouse TFF1 inactivation and the fully penetrant antropyloric tumor phenotype ${ }^{18}$ prompted the classification of TFF1 as a gastric tumor suppressor gene. Accordingly, altered expression, deletion, and/or mutations of the TFF1 gene have been observed in human gastric carcinomas. ${ }^{30,40-42}$ It has been shown that Cox-2 is expressed in TFF1 knockout adenomas, ${ }^{19}$ and this inverse relationship of COX-2 and TFF1 expression has been highlighted in our results, where loss of 
a
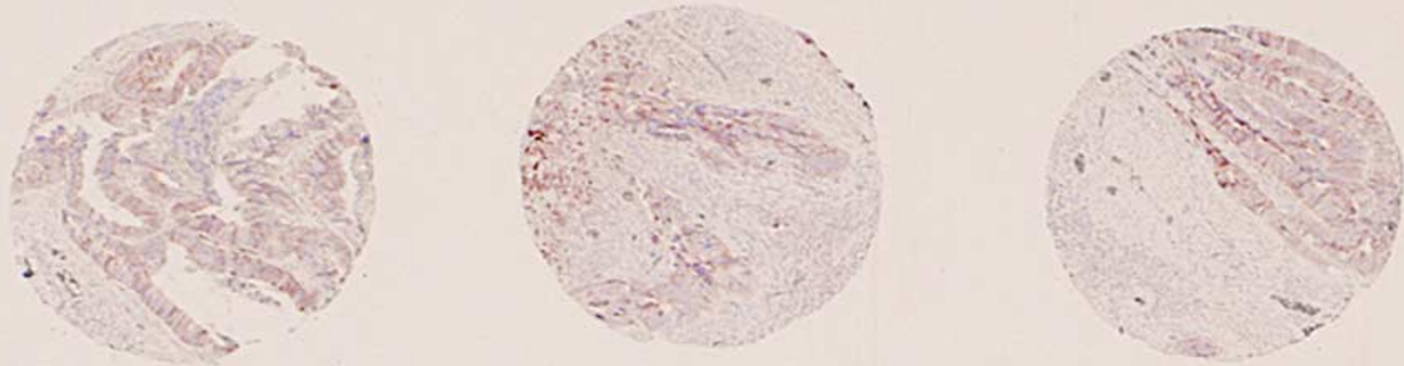

b
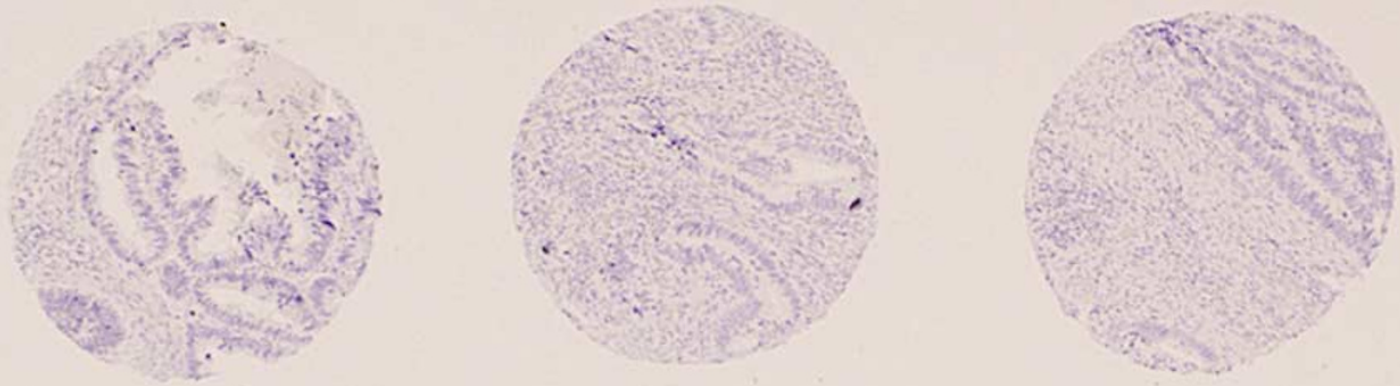

C

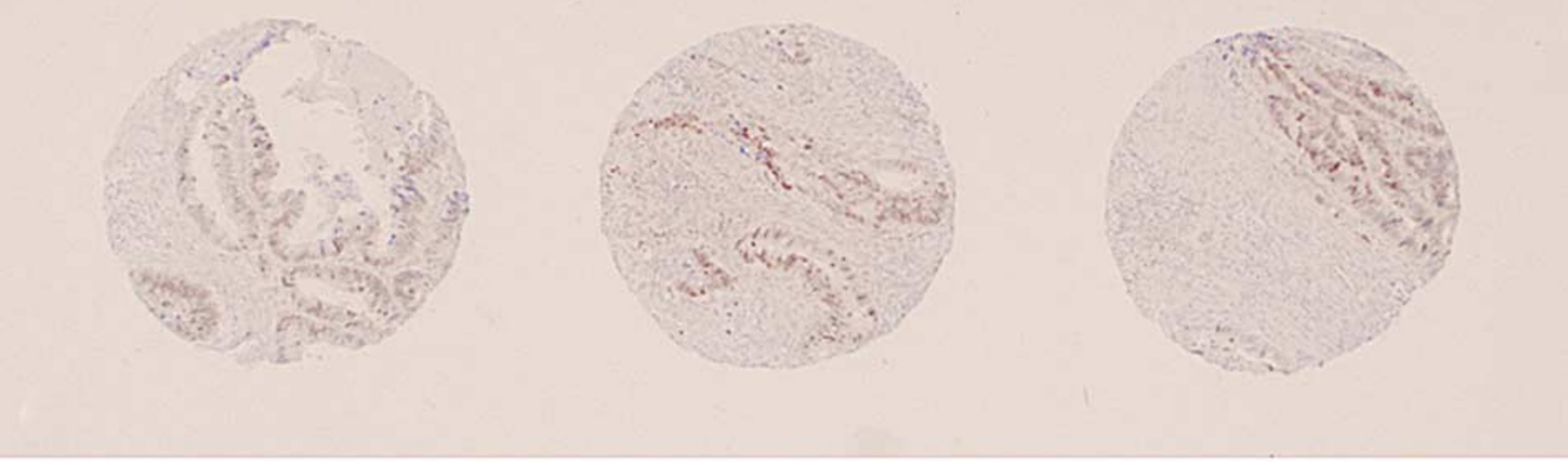

d
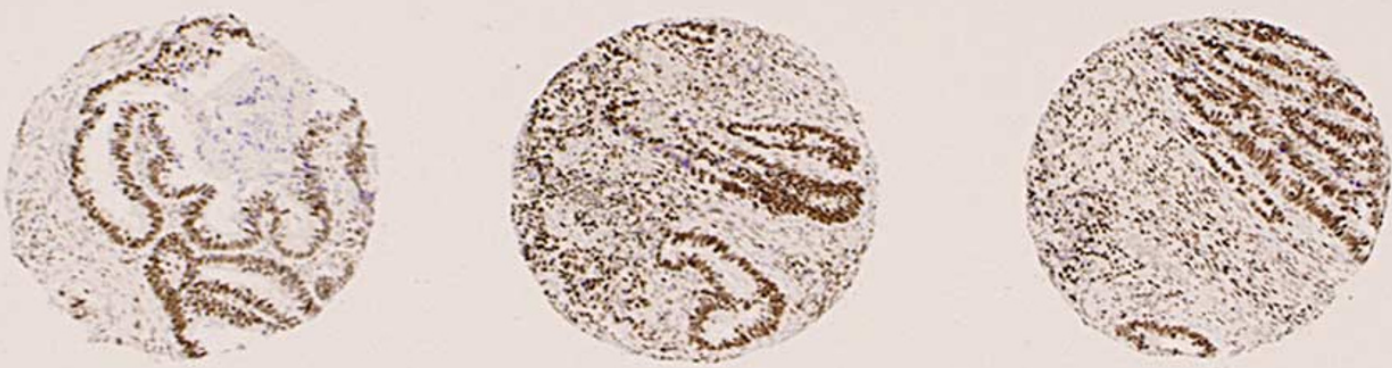

Figure 1 Immunohistochemistry on tissue microarrays for COX-2 (COX-2 High, a), TFF1 (negative, b), HuR (positive cytoplasmic staining, $\mathbf{c}$ ) and C/EBP- $\beta$ (positive, d). Magnification 32.5, counterstain hematoxylin. 
TFF1 and COX-2 High was seen in conventional tumors, whereas retention of TFF1 expression was more likely to pair with a COX-2 Low phenotype as seen in the early-onset gastric cancers.

Interestingly, it has been recently suggested that $\mathrm{C} / \mathrm{EBP}-\beta$, a transcription factor for $C O X-2,{ }^{43}$ plays a role in gastric cancer, through its relationship with $C O X-2 .{ }^{15}$ However, C/EBP- $\beta$ is also known to act as a transcription factor in the downregulation of TFF1, ${ }^{16,17}$ thus providing a fascinating link between COX-2 and TFF1, the two molecules shown here to be expressed differently in early onset gastric cancer and conventional gastric cancer. It raises the possibility that this one molecule could concomitantly downregulate TFF1, while upregulating COX-2, thus affecting two different carcinogenic pathways and providing a considerable advantage to tumor growth. In our study, the correlation between C/EBP- $\beta$ and TFF1 was only of borderline significance $(P=0.059)$ and thus the precise link between $\mathrm{C} / \mathrm{EBP}-\beta, \mathrm{COX}-2$ and TFF1 in gastric cancer needs further exploration.

This study also revealed molecular differences in histological type and anatomic location in gastric cancer. The significant differences found between cardia and body tumors in p53, c-jun and p16 expression lend further support to the theory that adenocarcinomas arising in the gastric cardia and body occur through different genetic pathways.

Despite the differences seen in COX-2 expression between early-onset gastric cancers and conventional cancers, and the possible crosstalk between the COX and Wnt pathways, ${ }^{24}$ no difference was seen in $\beta$-catenin, c-myc or c-jun expression between early-onset gastric cancer and conventional gastric cancer when using the binary logistic regression model. The well-established Wnt pathway is activated in colorectal cancer; however, not much is known about the relative importance of this pathway in gastric cancer and, in particular, early-onset gastric cancer. It has been found previously that immunohistochemical abnormalities of $\beta$-catenin are present in $22-27 \%$ of gastric cancer. ${ }^{44,45}$ Here, we confirm that estimate and show that although $\beta$ catenin may play an important role in gastric cancer, this role is not specific to early-onset gastric cancer. We also found no correlation between $\beta$-catenin and c-myc or c-jun, suggesting that these particular targets may not depend on activation of the Wnt pathway in gastric cancer.

$C$-myc is amplified in gastric cancer, and its amplification corresponds with c-myc overexpression on immunohistochemistry. ${ }^{46}$ The frequency of c-myc positivity found here is supported by previous findings where it was also found to be related to cell proliferation and associated with poor clinical outcome. ${ }^{35}$ Interestingly, we have found that nuclear HuR expression correlated with c-myc expression. HuR is known to stabilize c-myc mRNA in vitro, ${ }^{47}$ providing an explanation for the correla- tion between the two molecules, and suggesting for the first time that this interaction may be important in gastric carcinogenesis.

The proto-oncoprotein c-Jun is a component of the AP-1 transcription factor (a dimeric complex of Jun and Fos), the activity of which is augmented in many tumor types. The role of this transcription factor in gastric cancer is largely unknown. An important mechanism in the stimulation of AP-1 function is amino-terminal phosphorylation of c-Jun by the c-Jun N-terminal kinases (JNKs) and it has been shown that the phosphorylation-dependent interaction between c-Jun and TCF4 regulates intestinal tumorigenesis by integrating JNK and APC/beta-catenin, two distinct pathways activated by Wnt signalling. ${ }^{25}$ It has also been documented that a COX-2 inhibitor suppresses AP-1 through JNK in gastric cancer. ${ }^{48}$ In this study, we find c-jun positivity in a high percentage of gastric cancer and find that this positivity occurs more often in cancers of the stomach than in the cardia.

Finally, our findings with E-Cadherin, C/EBP- $\beta$, TFF1 and COX-2 expression emphasize the fact that diffuse and intestinal cancers differ at a molecular level. It has been suggested that loss of E-cadherin is the fundamental defect in diffuse-type gastric carcinoma, and that it provides an explanation for the observed morphological phenotype of noncohesive cells with loss of polarity and gland architecture. ${ }^{49,50}$ In contrast, gland architecture is preserved in the intestinal type of stomach cancer where loss of E-Cadherin expression does not occur as commonly. ${ }^{50}$ Here, we find that abnormal ECadherin does not associate directly with earlyonset gastric cancer if adjusted for histology, but instead correlates significantly with the diffuse phenotype. The converse has also been reported, ${ }^{51}$ although in the report by Lim et al, young cases were compared to old without adjusting for histology, perhaps explaining this discrepancy.

In summary, we have found further evidence that early-onset gastric cancer exhibits a unique expression profile of molecules important in carcinogenesis. This involves a COX-2 Low, TFF1-positive phenotype, which appears to occur almost exclusively in early-onset gastric cancer. It is interesting to note that these molecules are involved in inflammation, and C/EBP- $\beta$ and $\mathrm{HuR}$ appear to be involved in their regulation in gastric cancer. Our findings add further support to the hypothesis that young patients develop carcinomas with a different molecular genetic profile from that of sporadic carcinomas occurring at a later age.

\section{Acknowledgements}

This research was supported by a grant from the Vanderes Foundation. We thank Wilfred Meun for assistance with the figure. 


\section{References}

1 Pisani P, Parkin DM, Bray F, et al. Estimates of the worldwide mortality from 25 cancers in 1990. Int J Cancer 1999;83:18-29.

2 Laurén P. The Two histological main types of gastric carcinoma: diffuse and so-called intestinal-type carcinoma. An Attempt at a histo-clinical classification. Acta Pathol Microbiol Scand 1965;64:31-49.

3 Kokkola A, Sipponen P. Gastric carcinoma in young adults. Hepatogastroenterology 2001;48:1552-1555.

4 Kikuchi S, Nakajima T, Nishi T, et al. Association between family history and gastric carcinoma among young adults. Jpn J Cancer Res 1996;87:332-336.

5 Nomura A, Stemmermann GN, Chyou PH, et al. Helicobacter pylori infection and gastric carcinoma among Japanese Americans in Hawaii. N Engl J Med 1991;325:1132-1136.

6 Rugge M, Shiao YH, Busatto G, et al. The p53 gene in patients under the age of 40 with gastric cancer: mutation rates are low but are associated with a cardiac location. Mol Pathol 2000;53:207-210.

7 Parsonnet J, Vandersteen D, Goates J, et al. Helicobacter pylori infection in intestinal- and diffuse-type gastric adenocarcinomas. J Natl Cancer Inst 1991;83: 640-643.

8 Koshida Y, Koizumi W, Sasabe M, et al. Association of Helicobacter pylori-dependent gastritis with gastric carcinomas in young Japanese patients: histopathological comparison of diffuse and intestinal type cancer cases. Histopathology 2000;37:124-130.

9 Haruma K, Komoto K, Kamada T, et al. Helicobacter pylori infection is a major risk factor for gastric carcinoma in young patients. Scand J Gastroenterol 2000;35:255-259.

10 Guilford P, Hopkins J, Harraway J, et al. E-cadherin germline mutations in familial gastric cancer. Nature 1998;392:402-405.

11 Ristimaki A, Honkanen N, Jankala H, et al. Expression of cyclooxygenase-2 in human gastric carcinoma. Cancer Res 1997;57:1276-1280.

12 Saukkonen K, Rintahaka J, Sivula A, et al. Cyclooxygenase-2 and gastric carcinogenesis. Apmis 2003; 111:915-925.

13 Langman MJ, Cheng KK, Gilman EA, et al. Effect of anti-inflammatory drugs on overall risk of common cancer: case-control study in general practice research database. BMJ 2000;320:1642-1646.

14 Akre K, Ekstrom AM, Signorello LB, et al. Aspirin and risk for gastric cancer: a population-based case-control study in Sweden. Br J Cancer 2001;84:965-968.

15 Machado JC, Regalo G, Canedo P, et al. C/EBP-beta is expressed in gastric cancer and leads to overexpression of cycloxygenase-2. Proc Amer Assoc Cancer Res 2005;46:1934.

16 Dossinger V, Kayademir T, Blin N, et al. Downregulation of TFF expression in gastrointestinal cell lines by cytokines and nuclear factors. Cell Physiol Biochem 2002;12:197-206.

17 Sankpal NV, Mayo MW, Powell SM. Transcriptional repression of TFF1 in gastric epithelial cells by CCAAT/enhancer binding protein-beta. Biochim Biophys Acta 2005;1728:1-10.

18 Lefebvre O, Chenard MP, Masson R, et al. Gastric mucosa abnormalities and tumorigenesis in mice lacking the pS2 trefoil protein. Science 1996;274: 259-262.
19 Saukkonen K, Tomasetto C, Narko K, et al. Cyclooxygenase- 2 expression and effect of celecoxib in gastric adenomas of trefoil factor 1-deficient mice. Cancer Res 2003;63:3032-3036.

20 Shiao YH, Rugge M, Correa P, et al. p53 alteration in gastric precancerous lesions. Am J Pathol 1994;144: 511-517.

21 Leung WK, To KF, Ng YP, et al. Association between cyclo-oxygenase-2 overexpression and missense p53 mutations in gastric cancer. Br J Cancer 2001;84:335-339.

22 Shim YH, Kang GH, Ro JY. Correlation of p16 hypermethylation with p16 protein loss in sporadic gastric carcinomas. Lab Invest 2000;80:689-695.

23 Boon EM, Keller JJ, Wormhoudt TA, et al. Sulindac targets nuclear beta-catenin accumulation and Wnt signalling in adenomas of patients with familial adenomatous polyposis and in human colorectal cancer cell lines. Br J Cancer 2004;90:224-229.

24 Wang D, Mann JR, DuBois RN. WNT and cyclooxygenase-2 cross-talk accelerates adenoma growth. Cell Cycle 2004;3:1512-1515.

25 Nateri AS, Spencer-Dene B, Behrens A. Interaction of phosphorylated c-Jun with TCF4 regulates intestinal cancer development. Nature 2005;437:281-285.

26 Kononen J, Bubendorf L, Kallioniemi A, et al. Tissue microarrays for high-throughput molecular profiling of tumor specimens. Nat Med 1998;4:844-847.

27 Wang W, Furneaux $\mathrm{H}$, Cheng $\mathrm{H}$, et al. HuR regulates p21 mRNA stabilization by UV light. Mol Cell Biol 2000;20:760-769.

28 van Rees BP, Caspers E, zur Hausen A, et al. Different pattern of allelic loss in Epstein-Barr virus-positive gastric cancer with emphasis on the p53 tumor suppressor pathway. Am J Pathol 2002;161:1207-1213.

29 Carvalho R, Milne AN, Van Rees BP, et al. Early-onset gastric carcinomas display molecular characteristics distinct from gastric carcinomas occurring at a later age. J Pathol 2004;204:75-83.

30 Carvalho R, Kayademir T, Soares $\mathrm{P}$, et al. Loss of heterozygosity and promoter methylation, but not mutation, may underlie loss of TFF1 in gastric carcinoma. Lab Invest 2002;82:1319-1326.

31 Marsman WA, Birjmohun RS, van Rees BP, et al. Loss of heterozygosity and immunohistochemistry of adenocarcinomas of the esophagus and gastric cardia. Clin Cancer Res 2004;10:8479-8485.

32 Buskens CJ, Van Rees BP, Sivula A, et al. Prognostic significance of elevated cyclooxygenase 2 expression in patients with adenocarcinoma of the esophagus. Gastroenterology 2002;122:1800-1807.

33 de vos tot Nederveen Cappel WH, Offerhaus GJ, van Puijenbroek M, et al. Pancreatic carcinoma in carriers of a specific 19 base pair deletion of CDKN2A/p16 (p16-leiden). Clin Cancer Res 2003;9:3598-3605.

34 Erkinheimo TL, Lassus H, Sivula A, et al. Cytoplasmic HuR expression correlates with poor outcome and with cyclooxygenase 2 expression in serous ovarian carcinoma. Cancer Res 2003;63:7591-7594.

35 Han S, Kim HY, Park K, et al. c-Myc expression is related with cell proliferation and associated with poor clinical outcome in human gastric cancer. J Korean Med Sci 1999;14:526-530.

36 Brennan CM, Steitz JA. HuR and mRNA stability. Cell Mol Life Sci 2001;58:266-277.

37 Heinonen M, Bono P, Narko K, et al. Cytoplasmic HuR expression is a prognostic factor in invasive ductal breast carcinoma. Cancer Res 2005;65:2157-2161. 
38 Saukkonen K, Nieminen O, van Rees B, et al. Expression of cyclooxygenase-2 in dysplasia of the stomach and in intestinal-type gastric adenocarcinoma. Clin Cancer Res 2001;7:1923-1931.

39 van Rees BP, Saukkonen K, Ristimaki A, et al. Cyclooxygenase-2 expression during carcinogenesis in the human stomach. J Pathol 2002;196:171-179.

40 Beckler AD, Roche JK, Harper JC, et al. Decreased abundance of trefoil factor 1 transcript in the majority of gastric carcinomas. Cancer 2003;98:2184-2191.

41 Leung WK, Yu J, Chan FK, et al. Expression of trefoil peptides (TFF1, TFF2, and TFF3) in gastric carcinomas, intestinal metaplasia, and non-neoplastic gastric tissues. J Pathol 2002;197:582-588.

42 Park WS, Oh RR, Park JY, et al. Somatic mutations of the trefoil factor family 1 gene in gastric cancer. Gastroenterology 2000;119:691-698.

43 Caivano M, Gorgoni B, Cohen P, et al. The induction of cyclooxygenase-2 mRNA in macrophages is biphasic and requires both CCAAT enhancer-binding protein beta (C/EBP beta) and C/EBP delta transcription factors. J Biol Chem 2001;276:48693-48701.

44 Sasaki Y, Morimoto I, Kusano M, et al. Mutational analysis of the beta-catenin gene in gastric carcinomas. Tumour Biol 2001;22:123-130.
45 Woo DK, Kim HS, Lee HS, et al. Altered expression and mutation of beta-catenin gene in gastric carcinomas and cell lines. Int J Cancer 2001;95:108-113.

46 Kozma L, Kiss I, Hajdu J, et al. C-myc amplification and cluster analysis in human gastric carcinoma. Anticancer Res 2001;21:707-710.

47 Ma WJ, Cheng S, Campbell C, et al. Cloning and characterization of HuR, a ubiquitously expressed Elav-like protein. J Biol Chem 1996;271:8144-8151.

48 Wong BC, Jiang XH, Lin MC, et al. Cyclooxygenase-2 inhibitor (SC-236) suppresses activator protein-1 through c-Jun NH2-terminal kinase. Gastroenterology 2004;126:136-147.

49 Chan JK, Wong CS. Loss of E-cadherin is the fundamental defect in diffuse-type gastric carcinoma and infiltrating lobular carcinoma of the breast. Adv Anat Pathol 2001;8:165-172.

50 Machado JC, Soares P, Carneiro F, et al. E-cadherin gene mutations provide a genetic basis for the phenotypic divergence of mixed gastric carcinomas. Lab Invest 1999;79:459-465.

51 Lim S, Lee HS, Kim HS, et al. Alteration of E-cadherinmediated adhesion protein is common, but microsatellite instability is uncommon in young age gastric cancers. Histopathology 2003;42:128-136. 